of life is diminished, and a reduced sense of spiritual peace ensues. Yet many healthcare professionals feel unprepared to appreciate, assess and tackle patients' spiritual issues.

Cheshire \& Merseyside Palliative \& End of Life Care Network has run the 'Opening the Spiritual Gate' course, across the UK for a number of years, to address this training need. This aims to increase awareness of spiritual and religious needs and facilitate recognition of spiritual distress.

Aim To explore participant perceptions of spiritual care and the impact of the training on their clinical roles after completing the course.

Methods A qualitative methodology was adopted, using digitally recorded semi-structured interviews. Purposive sampling of healthcare professionals who had undertaken the course, in either the North West or South West of England between 2015-2017, resulted in 21 participants. Data were subject to thematic analysis.

Results All participants reported on the value of the course and the impact on their clinical roles, including being better able to recognise when spiritual distress may be evident. Two main themes were identified; recognising spirituality, containing sub-themes of what spirituality means and what matters, and supporting spiritual needs with sub-themes of recognition of spiritual distress, communication skills, not having the answers and going beyond the physical.

Conclusions The course is clearly effective in preparing staff to recognise opportunities to provide individualistic spiritual care. Acknowledging that spiritual care is as important as physical care, and having the skills to address it, is vital for delivery of best holistic care.

Funding Cheshire and Merseyside Palliative \& End of Life Network Education Strategy Group.

\section{P-164 USING VIRTUAL REALITY IN PATIENTS WITH PALLIATIVE CARE NEEDS}

Cecily Wright, Adarsh Babber, Sophie Price. Thames Hospice, Windsor, UK

\subsection{6/bmjspcare-2019-HUKNC.186}

Background Virtual Reality (VR) is a $360^{\circ}$ audio-visual simulation presented as reality to the user, allowing immersion and distraction from symptoms or treatments. There is some evidence based support for the use of VR technology to reduce symptoms including pain, anxiety and stress in patients with cancer, chronic pain and other conditions (Li, Montaño, Chen \& Gold, 2011; Gorini, Pallavicini, Algeri, Repetto et al., 2010; Oyama, 1998). The feasibility and impact of this technology was assessed in a hospice setting.

Method A pilot project for use of VR technology took place in a hospice inpatient and day unit over a four week period. Patients were assisted to use the technology by trained staff, with each session lasting approximately seven minutes. Patients chose their own 'experience' from a selection of options. A follow-up questionnaire on the experience, self-reported symptoms and any change in symptoms was completed by each patient.

Results A total of 14 sessions took place and each participant completed a questionnaire. All of the patients agreed they would use VR again and would recommend it to others. $75 \%$ of patients who reported pain felt they experienced some improvement following the session. Similarly, 50\% of patients reporting breathlessness and $81 \%$ expressing anxiety, believed they improved. Comments were generally positive, themed around 'immersion' and 'escapism'. The results were limited by the small number of participants and self-reported effect, but showed an overall improvement in symptoms.

Discussion This pilot study demonstrated VR technology to be a beneficial and well tolerated means of providing immersion and distraction therapy. Use was limited by the resources needed for the equipment, including cost, programmes available and trained staff. However, with rapidly emerging new technology and positive user feedback, VR is a viable noninvasive means for symptom management in palliative care.

\section{P-165 OBSERVATIONS OF IMMERSIVE VIRTUAL REALITY SESSIONS IN A HOSPICE}

Anna Lloyd, Erna Haraldsdottir. St Columba's Hospice, Edinburgh, UK

\subsection{6/bmjspcare-2019-HUKNC. 187}

Background Several studies have confirmed positive outcomes using virtual reality (VR) for clinical conditions such as anxiety disorders, phobias, post-traumatic stress syndrome, eating disorders and pain management. VR has also been employed to promote emotional wellbeing and induce positive emotions for people in hospital. Research into the clinical applicability of VR in health care settings is still in its infancy. Until now, little research has focused on VR in a hospice setting with one other hospice in the UK that has started a small research pilot. The aim of this study is to explore the feasibility, acceptability and potential benefits of using VR for people with incurable long term conditions in a hospice setting.

Methods This was a prospective observation and interview study of hospice patients' experiences of taking part in a 30 minute VR session where they were immersed in a virtual world.

Results Observational data of VR sessions with 20 patients showed that most people respond positively. Reactions were of joy, happiness and amazement or of feeling peaceful and calm. Some people voiced that they were able to forget symptoms. These participants valued being able to visit somewhere that they now never would while others were able to visit places, often from younger days, that they wished to see again, expressing that it allowed recourse to a happy time of life. Some participants' responses were more muted. Those participants would have preferred to have further opportunity to think about where they would like to visit having experienced a session. Some people, however, declined to take part stating that they saw no need for it, or that they felt that it was new and not for them.

Conclusion Preliminary findings are encouraging with VR well received by staff and patients alike. VR may best be targeted to people who are most likely to benefit from the technology.

\section{P-166 INTRODUCING ACUPUNCTURE AS A NON- PHARMACOLOGICAL OPTION FOR PALLIATIVE CARE SYMPTOMS IN THE HOSPICE}

Miriam Lemon. Wirral Hospice St John's, Higher Bebington, UK

\subsection{6/bmjspcare-2019-HUKNC. 188}

Background The evidence for acupuncture in a range of palliative symptoms is growing. Palliative patients are increasingly 